Journal of Advanced Research in Fluid Mechanics and Thermal Sciences

Journal homepage: www.akademiabaru.com/arfmts.html ISSN: 2289-7879

\title{
Preliminary Investigation of Using DBD Plasma for Application in Micro Combustors
}

\author{
Nurfarah Diana Mohd Ridzuan Tan ${ }^{1}$, Fudhail Abdul Munir ${ }^{1,2,}{ }^{,}$, Musthafah Mohd Tahir ${ }^{1,2}$, Herman \\ Saputro ${ }^{3}$, Masato Mikami ${ }^{4}$ \\ 1 Faculty of Mechanical Engineering, Universiti Teknikal Malaysia Melaka (UTeM), 76100 Durian Tunggal Melaka, Malaysia \\ 2 Centre for Advanced Research on Energy (CARe), Universiti Teknikal Malaysia Melaka (UTeM), 76100 Durian Tunggal Melaka, Malaysia \\ 3 Department of Mechanical Engineering Education, Universitas Sabelas Maret (UNS), Solo, Indonesia \\ 4 Department of Mechanical Engineering, Yamaguchi University of Japan, Japan
}

\section{ARTICLE INFO}

\section{Article history:}

Received 1 October 2020

Received in revised form 1 March 2021

Accepted 2 March 2021

Available online 14 April 2021

\section{ABSTRACT}

Keywords:

DBD plasma actuator; flow control; wind tunnel; micro combustion

\begin{abstract}
Dielectric-Barrier-Discharge (DBD) plasma actuators are one of the recent research topics that has caught worldwide attention. Plasma actuators are typically used in the aerospace field of study for their flow control and wide usage of different types of plasma actuators. DBD plasma actuator is an immobile actuator that able to be utilized for its flexibility and light weight parts. Due to the wide usage of DBD plasma, it is also able to be useful in the field of combustion in terms of air flow control. In this research, the DBD plasma actuator is tested on its ability to be applied in micro combustors based on the air flow controlling aspect, the temperature of plasma and effects of vibration of plasma. Experiments were performed in the wind tunnel with smoke generator to show the flow separation by DBD plasma actuator while infrared camera and accelerometer were used to sense the temperature and vibration respectively to investigate the effects of DBD plasma actuator on these aspects. Results shows that the plasma generated has minimal effects of the flow characteristics whereas the temperature of plasma has a maximum of $90^{\circ}$ celcius when it is generated continuously. The vibration results indicate that generating plasma produces a small amount of vibration.
\end{abstract}

\section{Introduction}

In recent decade, plasma actuators have become one of the increasing research topics that are researched on globally. One of the main usages are the flow control mechanism in plasma actuators that caught the attention of many researchers. The concept is such that plasma assisted combustion is capable of assisting ignition, flame stabilization and controlling the flow in aerodynamic and combustion. A controllable disturbance is created via plasma actuators that acts as a flow control by changing the current flow induced by the actuators. While plasma actuators bring many benefits in

\footnotetext{
* Corresponding author.

E-mail address: fudhail@utem.edu.my

https://doi.org/10.37934/arfmts.82.1.105112
} 
field research, it is also considered a very challenging topic to be applied in the field of combustion and aerodynamic in terms of flow control [1,2].

Combustion is a system which fuels are being burned in a chamber. Micro power generation is a system where it uses combustion as the base in a very small scale and is capable of producing a great amount of power. Micro combustion system uses heat produced from the chamber and converts the heat to electricity [3]. Typically, after the conversion of heat, the electricity is combined with microelectronic devices for convenience and portable [4]. Micro power generation system size scale characteristics is up to 1 millimeter size compared to its macro and meso scale system.

As in every research, there will always be difficulties and obstacles such as efficiency, emission rates, stability and heat resistance properties. Gaseous fuels were most convenient for micro combustion but depletion of natural resources forces the community to search different methods. Thus, bio-fuel such as liquid fuel was suggested. Liquid fuel is lighter causing it to be portable compared to gaseous fuel which are heavier. Now, with liquid fuel, electro-spraying method was introduced as the solution to the problem of using liquid fuel $[5,6]$. However, electro-spraying is complicated and requires specific materials use for the micro combustion. On the other hand, plasma actuator is lightweight, less complicated setup and can generated airflow to push liquid fuels towards the ignition chamber depending on the strength of the generated flow. So, this experimentation was done on plasma actuator to ensure that the parameters and conditions are reached so that it can be placed in a micro combustion for flow generation for liquid fuels without side effects such as unstable flow or unstable flame combustion.

There are many categories of plasma actuators which includes corona discharge, gliding arc plasma actuator and dielectric barrier discharge [7-11]. Dielectric Barrier Discharge (DBD) plasma actuator is one of the most popular plasma actuators that is singular and useful in many fields of research. It can be used in different ways and is less complex compared to other classes of plasma actuators. Plasma assisted combustions shows a noteworthy contribution in decreasing emission rate, increasing efficiency of combustion and improving the fuel reformation $[12,13]$.

Based on a few papers by He et al., [14], Daud et al., [15] and Sekimoto et al., [16], DBD plasma actuator is made of electrodes, a film such as polyimide tape (Kapton) acting as dielectric and has a high voltage power supply. Commonly, alternating current $(A C)$ are likely to be used as a power supply as compared to direct current (DC) with a range in $\mathrm{kV}$ and a frequency ranging $50 \mathrm{~Hz} \sim 50 \mathrm{kHz}$ [17]. Figure 1 shows the schematic of a singular DBD plasma actuator in a normal scenario.

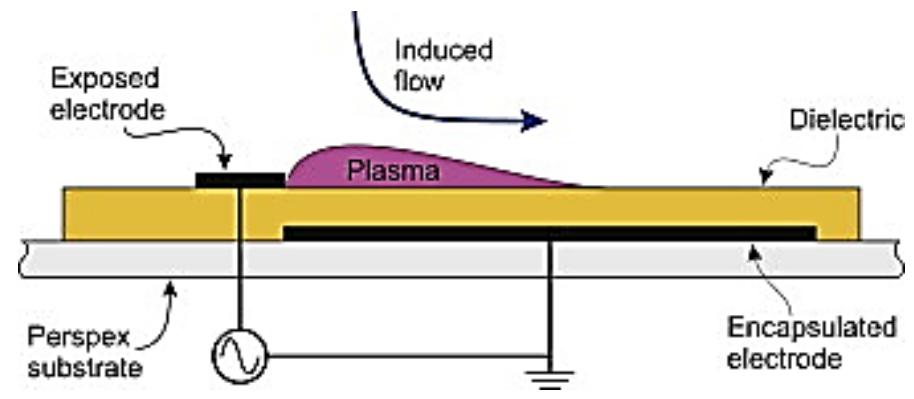

Fig. 1. Schematic of a Singular Dielectric-Barrier-Discharge (DBD) plasma actuator $[16,18]$

The studies on flow control of a DBD plasma actuator in terms of the characteristics of plasma assisted application are done in two ways, simulation and experimental studies. According to a paper that researched on both experimental and numerical investigation by Whalley and Choi [19], the visualization of the flow control field of the actuators shows a particular behavior proving that a DBD plasma actuator can entrained and quicken the surrounding fluid. A vortex or a spiral is formed and 
moving in a certain motion such as rolls up and then moving sideways away from the wall at specific angle. With longer time motion, a production of steady-state manner increases the momentum of the DBD plasma actuator.

Supporting the visualization results done by Whalley and Choi [19], the investigation in Kozato et al., [10] uses plasma to act as a spread controller on a rectangular jet by pulsing a modulated frequency as a means to control the flow of the plasma actuator. The method of using pulse modulated frequency as the controller is very effective and there is also vortex appearing. The vortex structure in this paper is affected by the frequency and the duty ratio while efficiency of flow control is based on the frequency.

Along that, the possibilities of using DBD plasma actuator was examined on flat and curved surfaces [20]. The design proposed are varying the shape and width of the electrodes of DBD plasma actuator. A micro actuator was fabricated to investigate the effect of generated airflow on an airfoil. Noteworthy, the curved micro DBD plasma actuator displays a better capability and produces a threedimensional (3D) flow structure compared to the straight DBD plasma actuator.

In terms of airflow assistance for DBD plasma actuators, the discharge generates a low velocity which can affect the airflow of the surroundings which in turn able to control that external airflow. Temperature is also one of the characteristics of plasma actuator where there is thermal (hot) and non-thermal (cold) plasma. DBD plasma is considered a cold plasma. Joussot et al., [21] uses an infrared camera to track the temperature of his plasma actuator in two environments; quiescent air and in influence of an external force. The paper concludes that external flow causes the temperature and flow to be disturbed. Compared to quiescent air, the condition with external force shows a decrease in dielectric temperature and indicates that heat dissipation occurs much better in a turbulent boundary layer than in laminar layer.

\section{Experimental Setup}

A model of DBD plasma actuator was designed and constructed using materials such as polyimide film (Kapton), copper tapes at $7 \mathrm{~mm}$ width and a voltage supply using an ozone generator system. A platform with a dimension of $135 \times 90 \times 25 \mathrm{~mm}$ for the DBD plasma actuator was fabricated using threedimensional (3D) printing machine. The DBD plasma actuator was placed on the fabricated platform to form a consistent data collection. The total length of DBD plasma actuator is $100 \mathrm{~mm}$ and was connected to the power supply via crocodile clip. The dielectric layer has a thickness of $0.06 \mu \mathrm{m}$ and there is a $0.05 \mathrm{~mm}$ streamwise gap between the copper electrodes. The power supply that drives the plasma actuator has an input of $220 \mathrm{~V}$ and an output of $6 \mathrm{kV}$ while producing $50 \mathrm{~Hz}$ of frequency.

To test the characteristics and capability of the generated plasma actuator, a wind tunnel with a smoke generator were used at different wind velocity which are $0 \mathrm{~m} / \mathrm{s}, 10 \mathrm{~m} / \mathrm{s}$ and $14 \mathrm{~m} / \mathrm{s}$ to facilitate the effect of plasma actuator on the drag coefficient produced. The drag coefficient is measured by using pressure sensors. A visual experiment using the smoke generator was tested on the plasma actuator. Figure 2 illustrates the DBD plasma actuator is placed on the fabricated platform while being mounted onto a stable position at $0^{\circ}$ angle of attack in the wind tunnel chamber.

The set-up for temperature testing includes an infrared camera and a computer. The image shown in Figure 3 is captured using a FLIR A615 thermal machine vision camera with a focal length $41.3 \mathrm{~mm}$. The distance of camera lens to the object (DBD plasma actuator) is set to $70 \mathrm{~mm}$ to get a good view of thermal changes of plasma. A software called FLIR Tools was used to examine the temperature maximum temperature generated by DBD plasma actuator.

In order to investigate the effect of plasma actuator on vibration, an accelerometer is used to test the vibration frequency produced by the plasma. This parameter used a $10 \mathrm{mV} / \mathrm{g}$ sensitivity 
accelerometer sensor on the platform near where the plasma was produced (see Figure 4). The program Vibration FFT Analysis software was used to capture the vibration at a sampling rate of $1 \mathrm{kS} / \mathrm{s}$ for a number of 1000 samples.

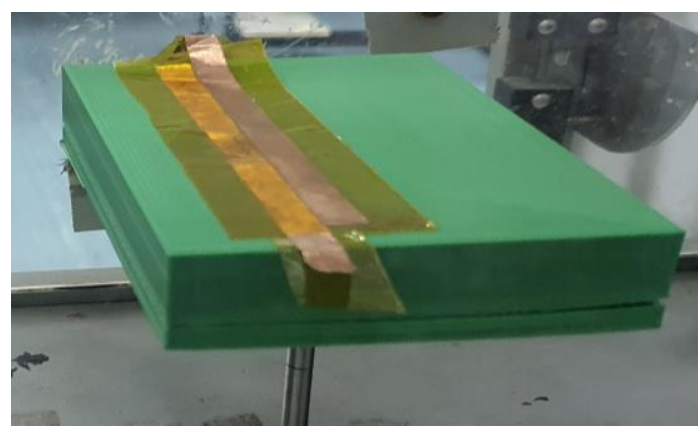

Fig. 2. DBD plasma on fabricated platform mounted in a wind tunnel chamber

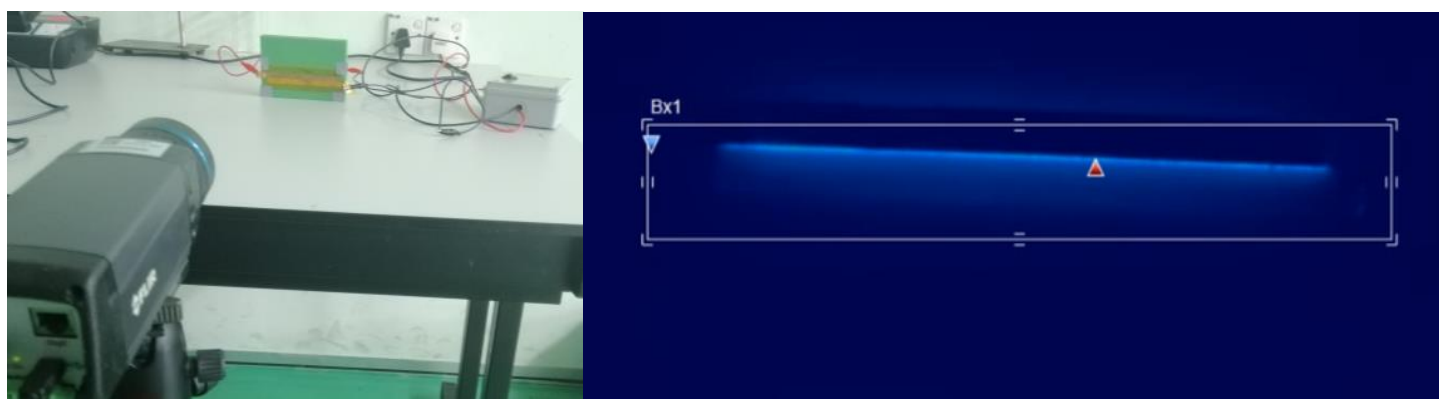

Fig. 3. Configuration to test Temperature of DBD Plasma Actuator

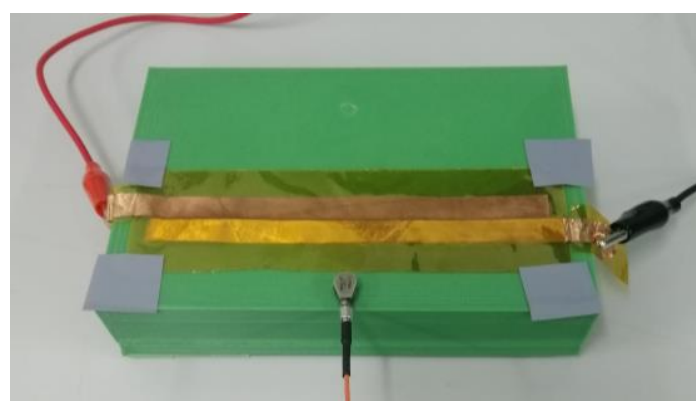

Fig. 4. Accelerometer is placed near generated DBD plasma actuator

\section{Results and Discussion}

\subsection{Pressure Distribution}

This section discusses the results obtained from the study of air flow, temperature and vibration characteristics of DBD plasma actuator. The characteristics of flow control, maximum temperature and vibration effect are discussed in the following sub section.

\subsection{Air Flow Characteristics via Wind Tunnel}

The test configuration for this experiment is in Table 1 where the properties are when the plasma is On and OFF to examine if plasma can disturb the flow in a closed chamber. Besides, a visual experimentation using smoke generator is performed showing minimal effect on the flow 
visualisation when the plasma actuator is turned on is illustrated in Figure 5 where there is minimal change in the flow of smoke. Following the speed of wind velocity, the smoke is generated when the velocity is set to $10 \mathrm{~m} / \mathrm{s}$ and $14 \mathrm{~m} / \mathrm{s}$.

Table 1

The values drag coefficient of DBD plasma

\begin{tabular}{llll}
\hline Properties & Wind Velocity & $\begin{array}{l}\text { Differential } \\
\text { Pressure } \\
\left(\mathrm{mmH}_{2} \mathrm{O}\right)\end{array}$ & $\begin{array}{l}\text { Drag } \\
\text { Coefficient at } \\
\left(0^{\circ}\right)\end{array}$ \\
\hline Plasma OFF & $0 \mathrm{~m} / \mathrm{s}$ & 47 & 0.00 \\
Plasma ON & $0 \mathrm{~m} / \mathrm{s}$ & 47 & 0.00 \\
Plasma OFF & $10 \mathrm{~m} / \mathrm{s}$ & 59 & 0.37 \\
Plasma ON & $10 \mathrm{~m} / \mathrm{s}$ & 59 & 0.36 \\
Plasma OFF & $14 \mathrm{~m} / \mathrm{s}$ & 72 & 0.65 \\
Plasma ON & $14 \mathrm{~m} / \mathrm{s}$ & 72 & 0.64 \\
\hline
\end{tabular}

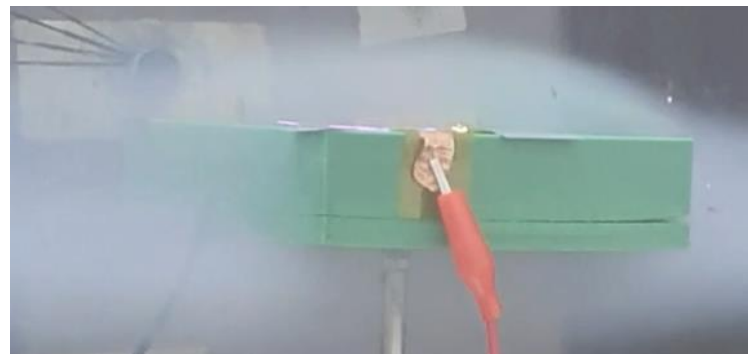

(a)

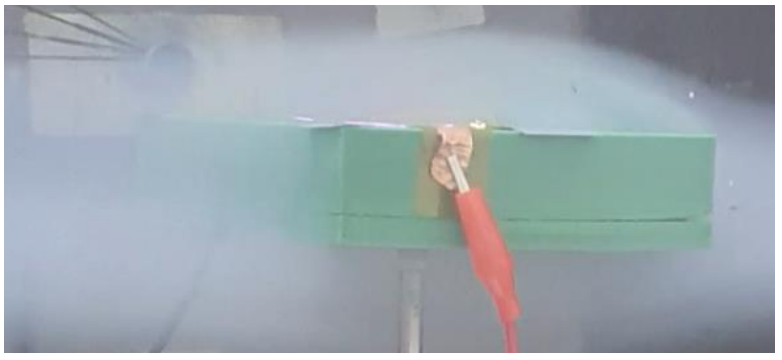

(b)

Fig. 5. Temperature image of DBD plasma actuator at (a) velocity $10 \mathrm{~m} / \mathrm{s},(\mathrm{b}) 14 \mathrm{~m} / \mathrm{s}$

The testing was done with smoke generator is to test the hypothesis made where plasma actuator generates a flow and a smoke screen is capable of visualizing the production of flow. In Table 1, there is only a minimal drag coefficient difference when the plasma actuator is turned ON and OFF. Theoretically, when DBD plasma actuator is turned ON, there is a flow disturbance occurring at the generation of plasma. The papers by Whalley and Choi [19] and Kozato et al., [10] show similar results in terms of effect of the flow control of DBD plasma actuator where there are vortex or compact spiral of smoke appeared when DBD plasma actuator is used. However, the preliminary experiment on the model in this study on visual flow field has no changes. One of the reasons is due to the fact that the voltage power supply is lacking to create a stronger plasma. The assumption that there is changes in visual observation of the plasma actuator is extremely minimal.

\subsection{Temperature of DBD Plasma Actuator}

A contactless thermal measurement is utilised in this experiment and to receive a clear image feedback from the computer, the distance of lens to the DBD plasma actuator is set to $700 \mathrm{~mm}$. The Figure 6 demonstrates the heat produced by the plasma actuator with the red arrow indicating the highest heat generation spot. The measurement range is from $-40-150{ }^{\circ} \mathrm{C}$ with a parameter of atmospheric temperate at $20^{\circ} \mathrm{C}$. 

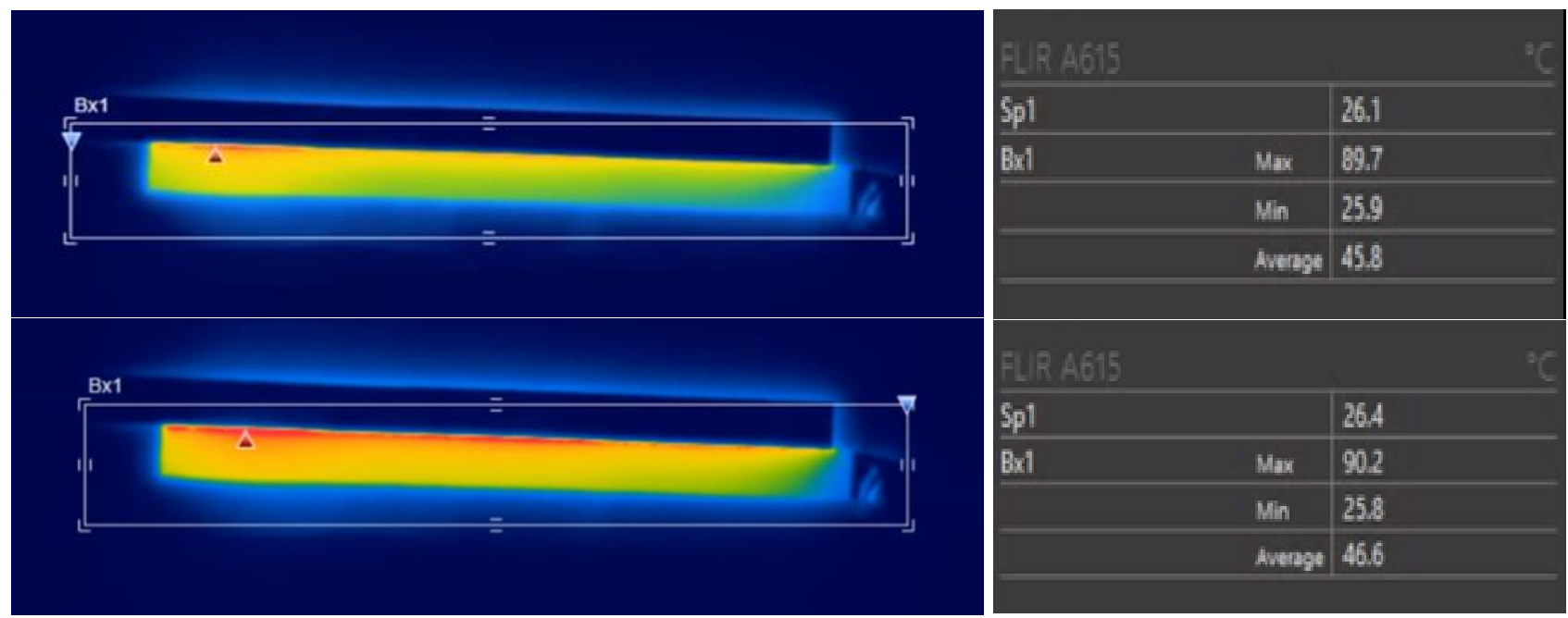

Fig. 6. Temperature image of DBD plasma actuator

Based on the results produced, and tested for a couple of times, the maximum temperature that is produced by the plasma actuator is $90^{\circ} \mathrm{C}$ at average. Repeated experiment was performed to ensure that the average accurate. Each experiment is timed to be within 1 minute and no longer due to the fact that plasma can corrode the dielectric layer when it is generated for a long period of time. The DBD plasma actuator also has a very short cooling time as when the plasma is switched OFF, the temperature rapidly cool down to room temperature within seconds. The temperature of DBD plasma actuator does play significant role in which it can heat the fuel slightly before entering the ignition phase in micro combustion whereby heated fuel can increase the efficiency of combustion. Here shows that DBD plasma actuator is able to influence the temperature of an experiment depending on the external factors such as laminar or boundary layer flows.

\subsection{Vibration Test Effect}

Vibration test effect were tested and observed due to vibrations can cause unstable ignition and combustion. This is because vibration can create an unsteady flow of fuel towards the ignition chamber if it has large vibration. Based on the experiment, two graphs were constructed to form a signal versus time graph and magnitude versus frequency graph. Figure 7 shows the graphs and signifies the maximum and minimum vibration output at $(0.5,0.07)$ and $(0.5,-0.06)$ respectively.

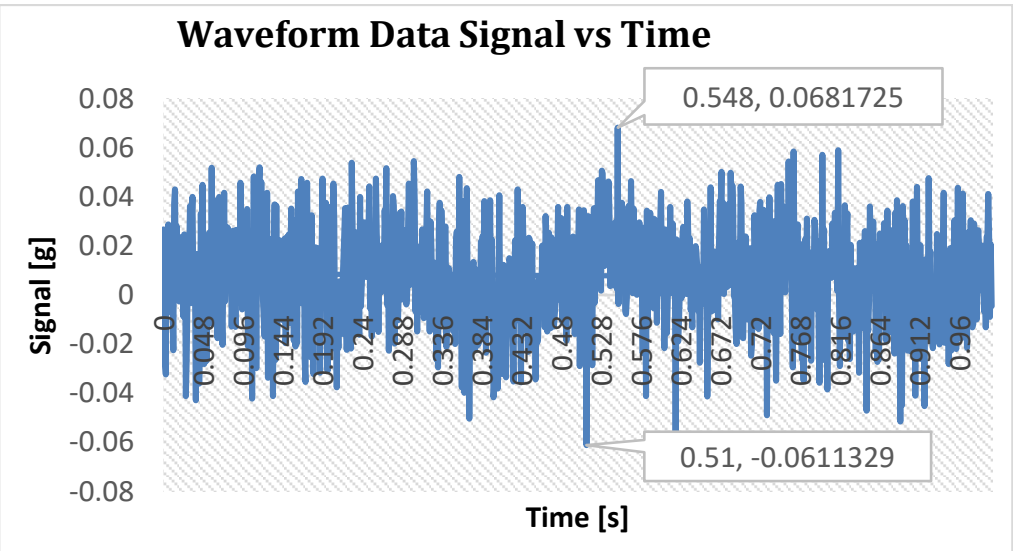

(a)

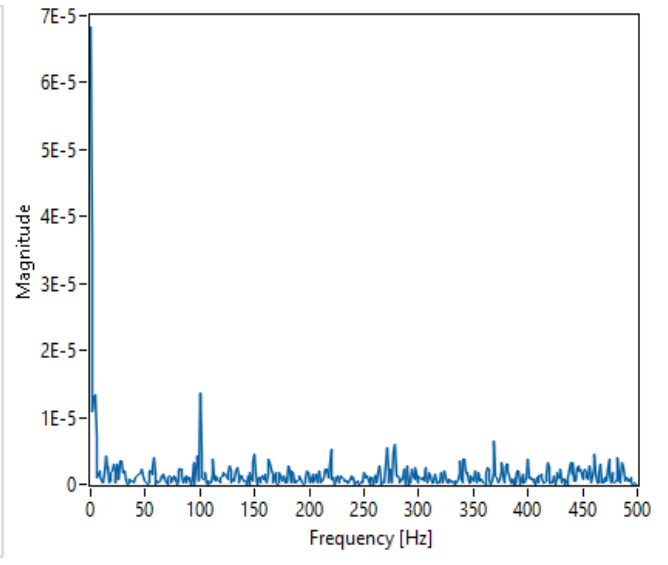

(b)

Fig. 7. Graph of vibration testing for (a) Signal vs Time and (b) Magnitude vs Frequency 
The results indicate that plasma that is generated is capable of emitting vibration. The vibration produced by the DBD plasma actuator is at bare minimum where it will not affect any experimentation data in many studies such as in combustion and micro combustion.

\section{Conclusions}

The data of DBD plasma actuator in three different characteristics is presented. The testing of hypothesis of flow visualisation of flow of plasma generation is done using smoke generator. Results illustrates there is a has minimal effects of the flow characteristics generated by the plasma in the wind tunnel while the temperature measured through contactless measurement shows that the temperature of plasma has a maximum of $90^{\circ}$ celcius when it is generated continuously. The vibration results indicate that generating plasma produces a minimal amount of vibration. As vibration and temperature of plasma is not high, the temperature and vibration generated is negligible and will not be able to influence the fuel or stability of combustion in micro combustors. Since this is a preliminary result to test the characteristics of DBD plasma actuator, more experiments need to be conducted for further research such as DBD plasma in smaller surface area as in cylindrical tube, curved surfaces and different sizes of plasma actuator in the cylindrical tube attached to the combustion chamber. The differences in combustion in different sizes of cylindrical chambers should also be investigated further.

\section{Acknowledgement}

This research was funded by a grant from Ministry of Higher Education of Malaysia (FRGS Grant FRGS/2018/FKM-CARe/F00370). The authors would like to thank Universiti Teknikal Malaysia Melaka (UTeM) for supporting this research activity.

\section{References}

[1] Go, David B. "Ionization and Ion Transport." A Primer for the Study of Non-Equilibrium, Low-Temperature Gas Discharges and Plas-mas (Morgan and Claypool Publishers, San Rafael, 2018) (2018). https://doi.org/10.1088/9781-6817-4601-2

[2] Tathiri, Gh, H. Parishani, S. G. Pouryoussefi, E. Esmaeilzadeh, S. M. Mirsajedi, M. Mirzaei, and N. Khatibzadeh. "Experimental Investigation of Separation Control on a NACA0024 Airfoil using Stationary and Non-Stationary ACDielectric Barrier Discharge Plasma Actuator." Journal of Applied Fluid Mechanics 9, no. 2 (2016): 877-888. https://doi.org/10.18869/acadpub.jafm.68.225.24432

[3] Fernandez-Pello, A. Carlos. "Micropower generation using combustion: Issues and approaches." Proceedings of The Combustion Institute 29, no. 1 (2002): 883-899. https://doi.org/10.1016/S1540-7489(02)80113-4

[4] Chou, S. K., W. M. Yang, K. J. Chua, J. Li, and K. L. Zhang. "Development of micro power generators-a review." Applied Energy 88, no. 1 (2011): 1-16. https://doi.org/10.1016/j.apenergy.2010.07.010

[5] Gan, Yunhua, Xiaowen Chen, Yang Tong, Xia Zhang, and Yang Zhang. "Thermal performance of a meso-scale combustor with electrospray technique using liquid ethanol as fuel." Applied Thermal Engineering 128 (2018): 274 281. https://doi.org/10.1016/j.applthermaleng.2017.09.016

[6] Yuliati, Lilis, Takehiko Seo, and Masato Mikami. "Liquid-fuel combustion in a narrow tube using an electrospray technique." Combustion and Flame 159, no. 1 (2012): $462-464$. https://doi.org/10.1016/i.combustflame.2011.06.010

[7] Labergue, A., E. Moreau, and G. Touchard. "A parametric study of surface corona discharge along an insulating flat plate in atmospheric pressure." In CEIDP'05. 2005 Annual Report Conference on Electrical Insulation and Dielectric Phenomena, 2005., pp. 490-494. IEEE, 2005.

[8] Feng, Rong, Jun Li, Yun Wu, Jiajian Zhu, Xiliang Song, and Xipeng Li. "Experimental investigation on gliding arc discharge plasma ignition and flame stabilization in scramjet combustor." Aerospace Science and Technology 79 (2018): 145-153. https://doi.org/10.1016/i.ast.2018.05.036 
[9] Lin, Bingxuan, Yun Wu, Yifei Zhu, Feilong Song, and Dongliang Bian. "Experimental investigation of gliding arc plasma fuel injector for ignition and extinction performance improvement." Applied Energy 235 (2019): 1017-1026. https://doi.org/10.1016/j.apenergy.2018.11.026

[10] Kozato, Yasuaki, Satoshi Kikuchi, Shigeki Imao, Yoshihisa Kato, and Katsumi Okayama. "Flow control of a rectangular jet by DBD plasma actuators." International Journal of Heat and Fluid Flow 62 (2016): 33-43. https://doi.org/10.1016/j.ijheatfluidflow.2016.09.014

[11] Tang, Yong, Jiankun Zhuo, Wei Cui, Shuiqing Li, and Qiang Yao. "Enhancing ignition and inhibiting extinction of methane diffusion flame by in situ fuel processing using dielectric-barrier-discharge plasma." Fuel Processing Technology 194 (2019): 106128. https://doi.org/10.1016/i.fuproc.2019.106128

[12] Sun, Wenting, Mruthunjaya Uddi, Timothy Ombrello, Sang Hee Won, Campbell Carter, and Yiguang Ju. "Effects of non-equilibrium plasma discharge on counterflow diffusion flame extinction." Proceedings of the Combustion Institute 33, no. 2 (2011): 3211-3218. https://doi.org/10.1016/i.proci.2010.06.148

[13] Starikovskiy, Andrey, and Nickolay Aleksandrov. "Plasma-assisted ignition and combustion." Progress in Energy and Combustion Science 39, no. 1 (2013): 61-110. https://doi.org/10.1016/i.pecs.2012.05.003

[14] He, Chuan, Thomas C. Corke, and Mehul P. Patel. "Plasma flaps and slats: an application of weakly ionized plasma actuators." Journal of Aircraft 46, no. 3 (2009): 864-873. https://doi.org/10.2514/1.38232

[15] Daud, Nazri Md, Yasuaki Kozato, Satoshi Kikuchi, and Shigeki Imao. "Control of leading edge separation on airfoil using DBD plasma actuator with signal amplitude modulation." Journal of Visualization 19, no. 1 (2016): 37-47. https://doi.org/10.1007/s12650-015-0283-0

[16] Sekimoto, Satoshi, Satoshi Shimomura, Hiroaki Fukumoto, Kozo Fujii, Akira Oyama, and Hiroyuki Nishida. "Experimental analysis of burst actuation for separation control around a pitching NACA0015 airfoil using a DBD plasma actuator at low Reynolds number." In 2018 AIAA Aerospace Sciences Meeting, p. 1551.2018. https://doi.org/10.2514/6.2018-1551

[17] Tathiri, Gh, E. Esmaeilzadeh, S. M. Mirsajedi, and H. Mahdavi Moghaddam. "Experimental Investigation of" Why an AC Dielectric Barrier Discharge Plasma Actuator is Preferred to DC Corona Wind Actuator in Boundary Layer Flow Control?"." Journal of Applied Fluid Mechanics 7, no. 3 (2014): 525-534. https://doi.org/10.36884/jafm.7.03.20378

[18] Erfani, Rasool, Tohid Erfani, Sergei V. Utyuzhnikov, and Konstantinos Kontis. "Optimisation of multiple encapsulated electrode plasma actuator." Aerospace Science and Technology 26, no. 1 (2013): 120-127. https://doi.org/10.1016/j.ast.2012.02.020

[19] Whalley, Richard D., and Kwing-So Choi. "The starting vortex in quiescent air induced by dielectric-barrier-discharge plasma." Journal of Fluid Mechanics 703 (2012): 192-203. https://doi.org/10.1017/jfm.2012.206

[20] Aono, Hikaru, Shota Yamakawa, Koh Iwamura, Shinji Honami, and Hitoshi Ishikawa. "Straight and curved type micro dielectric barrier discharge plasma actuators for active flow control." Experimental Thermal and Fluid Science 88 (2017): 16-23. https://doi.org/10.1016/i.expthermflusci.2017.05.005

[21] Joussot, Romain, Vincent Boucinha, Régine Weber-Rozenbaum, Hervé Rabat, Annie Leroy-Chesneau, and Dunpin Hong. "Thermal characterization of a DBD plasma actuator: dielectric temperature measurements using infrared thermography." In 40th Fluid Dynamics Conference and Exhibit, p. 5102. 2010. https://doi.org/10.2514/6.2010$\underline{5102}$ 